\title{
Catching the Sugars: Electrochemical Aptasensors for the Detection of Cancer-Related Glycosylation Changes in Prostate Specific Antigen +
}

\author{
Ana Díaz-Fernández 1,2,*, Rebeca Miranda-Castro ${ }^{1,2}$, Pedro Estrela ${ }^{3,4}$, \\ Noemí de-los-Santos-Álvarez ${ }^{1,2}$ and María Jesús Lobo-Castañón 1,2 \\ 1 Departamento de Química Física y Analítica, Universidad de Oviedo, Av. Julián Clavería 8, 33006 Oviedo, \\ Spain; mirandarebeca@uniovi.es (R.M.-C.); santosnoemi@uniovi.es (N.d.-1.-S.-Á.); \\ mjlc@uniovi.es (M.J.L.-C.) \\ 2 Instituto de Investigación Sanitaria del Principado de Asturias, Av. de Roma, 33011 Oviedo, Spain \\ 3 Centre for Biosensors, Bioelectronics and Biodevices (C3Bio), University of Bath, Bath BA2 7AY, UK; \\ P.Estrela@bath.ac.uk \\ 4 Department of Electronic and Electrical Engineering, University of Bath, Bath BA2 7AY, UK \\ * Correspondence: anadfdez@gmail.com \\ † Presented at the 1st International Electronic Conference on Biosensors, 2-17 November 2020; Available \\ online: https://iecb2020.sciforum.net/.
}

Received: date; Accepted: date; Published: date

\begin{abstract}
Prostate Specific Antigen (PSA) is the biomarker used for prostate cancer (PCa) detection although its lack of specificity results in high rate of false-positives and many unnecessary biopsies. Therefore, there is a need for more specific cancer biomarkers for PCa. Recent studies have shown that aberrant glycosylation of proteins is a common feature of the presence of cancer. In the case of prostate cancer, there are changes in core-fucose and sialic acids in the glycan structure of PSA. In this work, we describe two different strategies to direct the selection of aptamers toward the glycans of PSA. From these strategies we identified two aptamers (PSA-1 and PSAG-1) that bind to the glycan structure of PSA with high affinity. Both aptamers are applied in the design of electrochemical aptasensors, sandwich and direct formats, to detect the changes in the glycosylation of PSA. The sensors respond to different levels of PSA in serum and they show higher potential to discriminate clinically meaningful PCa than ELISA test used in hospitals (reducing the number of false positive), although validation on more samples is needed.
\end{abstract}

Keywords: Prostate Specific Antigen; SELEX; aptamers; electrochemical aptasensors; glycoproteins; prostate cancer

\section{Introduction}

Prostate cancer (PCa) is the most frequently diagnosed cancer and the second cause of cancer death in men. The approved tests to diagnose prostate cancer are digital rectal exam and the analysis of the levels of Prostate Specific Antigen (PSA) in blood. However, because their low sensitivity and specificity, an invasive prostate biopsy is needed to confirm the presence of the tumor. PSA is the most prevalently used PCa biomarker in the clinical practice. The test consists in the measurement of PSA levels in serum, with a cut-off of $4 \mathrm{ng} / \mathrm{mL}$. But this test is not specific in the gray zone, between 4 and $10 \mathrm{ng} / \mathrm{mL}$, where the concentration of PSA is not only indicative of cancer but also of other benign prostate diseases. This results in a high number of false positives and leads to many unnecessary biopsies. Therefore, new tests for PCa diagnosis are needed [1,2]. 
The well-established association between aberrant glycosylation and cancer suggests that the analysis of tumor-linked glycan alterations could be a valuable tool for cancer diagnosis [3]. In prostate cancer, a variation in the content of the fucose and the sialic acids in the glycan linked to PSA was described [4]. The glycosylation profile is currently characterized by long and complicated protocols, based on final mass spectrometry detection or the use of lectins. For routine analysis, however, the development of rapid direct methods for detecting aberrant glycosylated forms of PSA requires specific receptors with higher affinity than lectins. Aptamers can be tailored to specifically recognize the glycan moiety turning into promising synthetic receptors to replace the current methods for glycosylation profiling [5].

In this work, we present a strategy to select aptamers toward the glycosylation site of the glycoprotein PSA. The aptamers were used in the design of electrochemical aptasensors for the detection of changes in the glycosylation pattern of PSA associated to PCa (Figure 1).
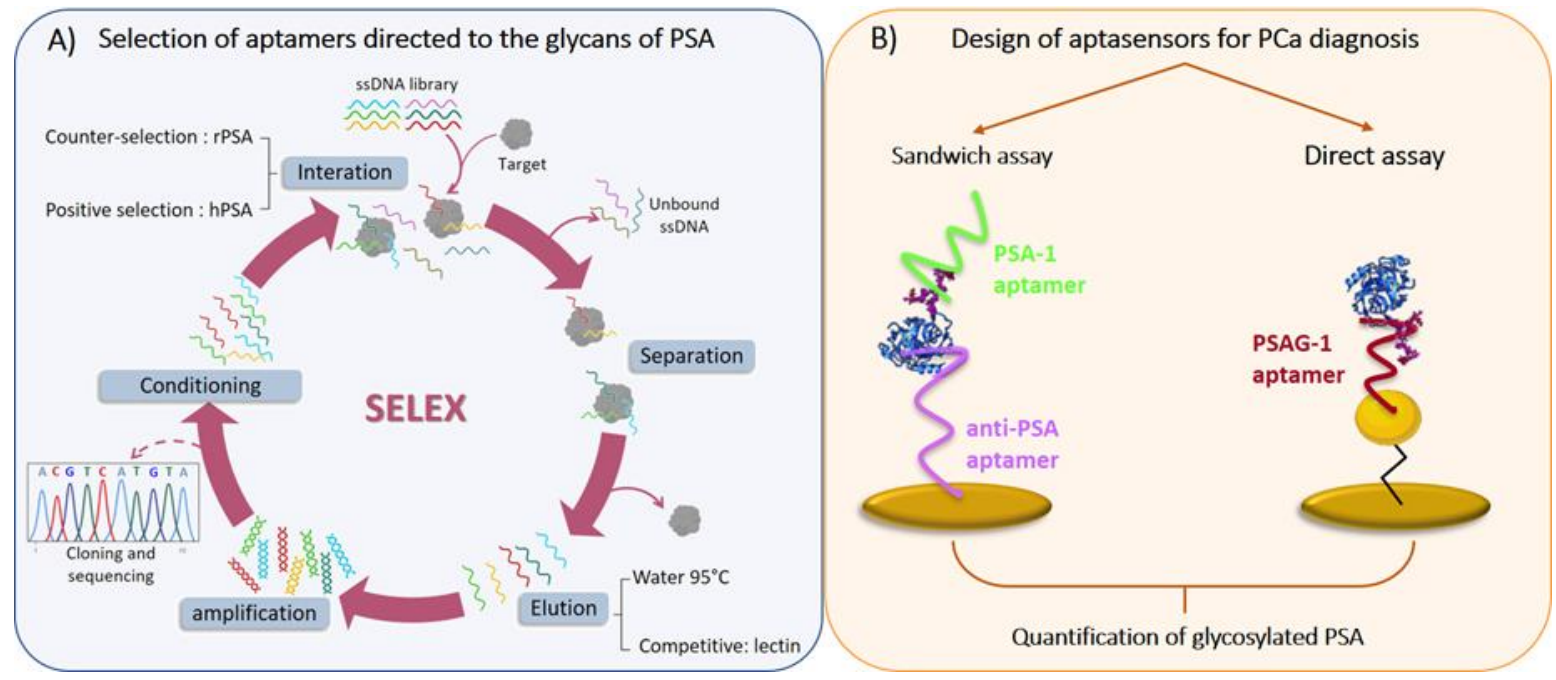

Figure 1. Overview of the work described in this conference proceeding paper: (A) selection of aptamers directed to the glycan structure of PSA and (B) design of aptasensors for prostate cancer diagnosis.

\section{Materials and Methods}

\subsection{Materials and Apparatus}

Detailed list of the materials and apparatus is available in references [6,7].

\subsection{SELEX Procedure}

SELEX procedure is an iterative process, and each cycle consists of the following steps: incubation of a ssDNA library with the target (PSA), separation of the unbound sequences, elution of the bound ones, amplification of the eluted sequences by PCR and strand separation to start a new round of selection (Figure 1).

In this work we used an initial library of ssDNA 80 nucleotides (nt)-length with a $40 \mathrm{nt}$ randomized central region flanked at both ends by two constant sequences of 20 nucleotides each. To make easier the separation and elution steps the protein PSA was immobilized on the surface of tosylactivated magnetic particles. To direct the selection toward the glycosylation site of the protein we performed two strategies: one is based on the introduction of counter-selection steps with recombinant PSA (rPSA), an unglycosylated form of the protein, and the second one is based on counter-selection with rPSA and competitive elution with a lectin.

The counter-selection step consisted in the incubation of the ssDNA library with rPSA, and after the interaction the unbound sequences were collected and incubated with PSA. The following steps of the SELEX were performed in the traditional way. 
The competitive elution with the lectin consisted in the elution of the sequences bound to PSA by adding a high concentration of the lectin in solution, which displaced the sequences that binds to the lectin-binding site.

A comprehensive and detailed explanation of the protocols could be found in reference [6,7].

\subsection{Electrochemical Binding Assays}

Aptamers were screened by affinity assays with electrochemical detection. With this aim, PSA was covalently immobilized onto screen-printed gold electrodes. First, a self-assembled monolayer (SAM) of a mixture 1:3 of 11-mercaptoundecanoic acid and 6-mercaptohexanol (both $1 \mathrm{mM}$ ) in NaAc buffer $\left(10 \mathrm{mM} \mathrm{pH} \mathrm{5.5)}\right.$ was prepared overnight at $4{ }^{\circ} \mathrm{C}$. The carboxylic groups were activated with a solution of $100 \mathrm{mM}$ EDC and $25 \mathrm{mM}$ NHS in water for $30 \mathrm{~min}$ and then PSA was covalently immobilized by incubating the electrodes in $50 \mu \mathrm{g} / \mathrm{mL}$ of PSA for $30 \mathrm{~min}$. Finally, the remaining active groups were blocked with ethanolamine $1 \mathrm{M}$ in PBS $(1 \times \mathrm{pH} 7.4)$ for $30 \mathrm{~min}$.

Binding assays were performed by incubating the modified electrode with increasing concentrations of the aptamers labeled with fluorescein for $30 \mathrm{~min}$ in PBS. After the interaction, the conjugate Fab-antifluorescein-peroxidase (Fab-POD: $0.5 \mathrm{U} / \mathrm{mL}$ in PBS-0.5\% casein) was added for 30 min. Finally, the electrochemical measurement was carried out with $35 \mu \mathrm{L}$ of $\mathrm{TMB}-\mathrm{H}_{2} \mathrm{O}_{2}$ and the enzymatic product was measured after $30 \mathrm{~s}$ by chronoamperometry at $-0.2 \mathrm{~V}$ for $1 \mathrm{~min}$. The current obtained during the last $10 \mathrm{~s}$ was averaged and related with the aptamer concentration.

\subsection{Deglycosyation Studies}

The interaction of PSA with specific glycosidase enzymes was performed on screen-printed gold electrodes modified with PSA as indicated above. The incubation order was as follows: neuraminidase- $\mathrm{A}, \beta$-galactosidase, $\mathrm{N}$-acetylhexosaminidase, mannosidase, fucosidase and PNGaseF. The preparation of the enzymes (buffer and concentration) was performed following the manufacturer's protocols. The incubations were performed at $37^{\circ} \mathrm{C}$ in a volume of $10 \mu \mathrm{L}$ in a wet atmosphere for $2 \mathrm{~h}$, except for the PNGaseF enzyme with an incubation period of $24 \mathrm{~h}$. Then, the binding capacity of the aptamers was tested performing an electrochemical binding assay with PSAG-1 or PSA-1 as indicated above [7].

\subsection{Sandwich Aptasensor}

To fabricate the sensor, the capture biotinylated aptamer (anti-PSA) was immobilized onto screen-printed gold electrodes modified with streptavidin. For PSA analysis, different concentrations of PSA prepared in TBS (10 mM Tris- $\mathrm{HCl}, 150 \mathrm{mM} \mathrm{NaCl}, 5 \mathrm{mM} \mathrm{KCl}, 5 \mathrm{mM} \mathrm{MgCl} 2 \mathrm{pH} 7.4)$ or diluted serum were incubated on the working electrode for $30 \mathrm{~min}$ and then the detection aptamer (PSA-1) was added for $30 \mathrm{~min}$. Next, the bound aptamer is labeled with Fab-POD for $30 \mathrm{~min}$ in TBS-0.5\% casein. Finally, the electrochemical measurement was carried out as described above [6].

\subsection{Impedimetric Aptasensor}

A dual impedimetric platform for direct measurement of PSA was developed by immobilizing a thiolated aptamer (anti-PSA or PSAG-1) onto nanostructured gold electrodes. Each aptamer layer was prepared onto gold electrodes modified with 11-amino-1-undecanothiol and 6-mercapto-1hexanol and attaching gold nanoparticles to the amine group in the SAM. For the PSA detection, the sensors were incubated with increasing concentrations of PSA in PBS for $30 \mathrm{~min}$ and, after the interaction, the impedance was measured in $5 \mathrm{mM} \mathrm{Fe}\left[(\mathrm{CN})_{6}\right]^{3-/ 4-}$ at $0.22 \mathrm{~V}$ vs. $\mathrm{Ag} \mid \mathrm{AgCl}, \mathrm{KCl}$ $(3 \mathrm{M}) \mid \mathrm{KNO}_{3}(3 \mathrm{M})$. 


\section{Results and Discussion}

\subsection{Selection and Characterization of Aptamers}

To direct the selection of aptamers toward the glycosylation site of PSA two counter-selection steps against the recombinant PSA (rPSA) were introduced into six-round SELEX. One of the obtained aptamers (PSA-1) showed preferential binding for PSA over rPSA and its affinity and selectivity was evaluated in detail. A dissociation constant of $357 \pm 42 \mathrm{nM}$ was estimated. However, this aptamer also recognized other glycoprotein (Lipocalin-2 or NGAL), meaning that PSA-1 binds to the glycan moiety and not to the peptide region of the protein. In consequence, PSA- 1 might be a good receptor for human glycans [6].

To refine the selection of aptamers, obtaining receptors able to differentiate glycoproteins with similar glycosylation patterns we proposed a selection based on counter-selection steps against rPSA and competitive elution with the lectin PhoSL (specific binder of $\alpha 1-6$ core-fucose). After ten rounds of selection, one aptamer (PSAG-1) preferentially bound to PSA with a dissociation constant of $34 \pm$ $9 \mathrm{nM}$. This aptamer showed selectivity against other glycoproteins: NGAL, AFP, ovalbumin and transferrin. This indicates that the molecular interaction of PSAG-1 and PSA involved both the glycans and some amino acids of the protein [7].

\subsection{Study of the Binding Site}

To find out which sugars in the glycan structure are involved in the recognition of PSA by both aptamers the sugars of PSA immobilized onto gold electrodes were removed, using selective glycosidases. After each sugar removal, the binding ability of the aptamers was tested. From this study we observed that PSA-1 and PSAG-1 aptamers have a completely different binding pattern. PSA-1 recognizes the external sugars (sialic acid and galactose) while PSAG-1 recognizes the innermost sugars [7].

\subsection{Detection of Glycosylated PSA Using Aptasensors}

We employed the selected aptamers and an aptamer previously described (anti-PSA), which only recognizes the peptide moiety [8], for the development of electrochemical aptasensors. Two different formats: sandwich and direct label-free approaches were designed using gold electrodes as support.

The sandwich aptasensor, using anti-PSA as the capture aptamer and PSA-1 as the detection aptamer, has a working range between $0.66 \mathrm{ng} / \mathrm{mL}$ and $25 \mathrm{ng} / \mathrm{mL}$ in 1:1 diluted serum, and a detection limit of $0.66 \mathrm{ng} / \mathrm{mL}$ of PSA. This sensor has an excellent selectivity against other proteins (rPSA and NGAL) and 92 analytes present in biological fluids [6].

The direct label-free assay was performed on a dual impedimetric platform with two sensors, one with anti-PSA and the other with PSAG-1 aptamer. Both sensors respond to increasing concentrations of PSA in 1:5 diluted serum. The working range of the anti-PSA sensor is between 0.64 $\mathrm{ng} / \mathrm{mL}$ and $62.5 \mathrm{ng} / \mathrm{mL}$, and $0.26 \mathrm{ng} / \mathrm{mL}$ to $62.5 \mathrm{ng} / \mathrm{mL}$ for the PSAG-1 sensor [9].

Both aptasensors were applied to the analysis of serum samples from patients with benign or malignant prostate pathologies from the clinical analysis laboratory at Hospital de Cabueñes (Gijón, Spain). For samples with PSA concentrations in the grey zone or above, the aptasensors provided discordant values with those obtained by the ELISA but in close agreement with the final diagnosis (no prostate cancer). Therefore, the results point to the detection of glycans as an alternative for the detection of prostate cancer, with potential to improve the clinical outcomes of current PSA test, reducing the number of unnecessary biopsies [6,9].

Funding: This research was funded by the Spanish Government (project RTI-2018-095756-B-I00) and Principado de Asturias Government (IDI2018-000217), co-financed by FEDER funds. A.D-F. thanks Asociación Española Contra el Cáncer (AECC) for her predoctoral grant and Banco Santander and University of Oviedo for the grant in the framework of the economic mobility of excellence for teachers and researchers.

Acknowledgments: We thank E. Fernández-Rodríguez and S. García-Alonso (Hospital Universitario de Cabueñes-Asturias) for providing the serum samples. 
Conflicts of Interest: The authors declare no conflict of interest.

\section{References}

1. Lilja, H.; Ulmert, D.; Vickers, A.J. Prostate-specific antigen and prostate cancer: Prediction, detection and monitoring. Nat. Rev. Cancer 2008, 8, 268-278, doi:10.1038/nrc2351.

2. Lomas, D.J.; Ahmed, H.U. All change in the prostate cancer diagnostic pathway. Nat. Rev. Clin. Oncol. 2020, 17, 372-381, doi:10.1038/s41571-020-0332-z.

3. Pinho, S.S.; Reis, C.A. Glycosylation in cancer: Mechanisms and clinical implications. Nat. Rev. Cancer 2015, 15, 540-555, doi:10.1038/nrc3982.

4. Gilgunn, S.; Conroy, P.J.; Saldova, R.; Rudd, P.M.; O'Kennedy, R.J. Aberrant PSA glycosylation-A sweet predictor of prostate cancer. Nat. Rev. Urol. 2013, 10, 99-107, doi:10.1038/nrurol.2012.258.

5. Díaz-Fernández, A.; Miranda-Castro, R.; de-los-Santos-Álvarez, N.; Lobo-Castañón, M.J. Post-translational modifications in tumor biomarkers: The next challenge for aptamers? Anal. Bioanal. Chem. 2018, 410, 2059 2065, doi:10.1007/s00216-018-0861-9.

6. Díaz-Fernández, A.; Miranda-Castro, R.; de-los-Santos-Álvarez, N.; Fernández-Rodríguez, E.; LoboCastañón, M.J. Focusing aptamer selection on the glycan structure of prostate-specific antigen: Toward more specific detection of prostate cancer. Biosens. Bioelectron. 2019, 128, 83-90, doi:10.1016/j.bios.2018.12.040.

7. Díaz-Fernández, A.; Miranda-Castro, R.; Díaz, N.; Suárez, D.; de-los-Santos-Álvarez, N.; Lobo-Castañón, M.J. Aptamers targeting protein-specific glycosylation in tumor biomarkers: General selection, characterization and structural modeling. Chem. Sci. 2020, 11, 9402-9413, doi:10.1039/d0sc00209g.

8. Savory, N.; Abe, K.; Sode, K.; Ikebukuro, K. Selection of DNA aptamer against prostate specific antigen using a genetic algorithm and application to sensing. Biosens. Bioelectron. 2010, 26, 1386-1391, doi:10.1016/j.bios.2010.07.057.

9. Díaz-Fernández, A.; Miranda-Castro, R.; de-los-Santos-Álvarez, N.; Lobo-Castañón, M.J.; Estrela, P. Impedimetric aptamer-based glycan PSA score for discrimination of prostate cancer from other prostate diseases. Biosens. Bioelectron. 2020, submitted.

Publisher's Note: MDPI stays neutral with regard to jurisdictional claims in published maps and institutional affiliations.

(C) 2020 by the authors. Submitted for possible open access publication under the terms and conditions of the Creative Commons Attribution (CC BY) license (http://creativecommons.org/licenses/by/4.0/). 